\title{
The framing of decision making support systems on increasing community resilience in disaster risk reduction efforts : a conceptual approach
}

\author{
Putranesia Thaha $^{1 *}$ and Febrin Anas Ismail $^{2}$ \\ ${ }^{1}$ Dept. of Quantity Surveying, Faculty of Engineering and Planning, Bung Hatta University, Indonesia \\ ${ }^{2}$ Dept. of Civil Engineering, Faculty of Engineering, Andalas University, Indonesia.
}

\begin{abstract}
This research begins by comprehensively exploring previous research related to community resilience and what steps are used to increase community resilience in reducing disaster risk. Conceptually, it is known that the fatigue model accumulated by the time system, infrastructure system, governance system, regulatory system, and hazard system for disaster risk reduction is often associated with weakening community resilience. It is often associated with catastrophic events, which are sometimes predictable and unpredictable. In manual decision-making, people are aware of the inconsistency of subjective decisions. A decision support system hypothesizes that it will take less time to explore data to make faster and more informed decisions. As a result of this concept, it is possible to reduce the number of wrong choices when dealing with disaster risk reduction issues. In terms of disaster risk reduction, the power of decision support systems is discussed in this paper to find a framework for its effectiveness as relative decision making will differ on different dimensions of Resilience.
\end{abstract}

\section{Introduction}

Resilience can be described as overcoming obstacles and reducing the duration of deviations from the level of system performance and Resilience to control the difficulties encountered in life [1], [2]. Meanwhile, capability can be interpreted as a resource which is a capability, and capacity as an essential component that can affect the results or goals [3]. Furthermore, Rus, Kilar, and Koren. [4], identified Resilience as two dominant theoretical perspectives, namely socio-ecology and engineering; the socio-ecological perspective is considered a process-oriented phenomenon (a dynamic concept), while the technical perspective views Resilience as a result-or-concept (static premise).

In line with the socio-ecological philosophy above, Davies [5] said that disaster resilience could be increased when the social impact of disasters is reduced. In terms of reducing the effects of the disaster, planning for Resilience as a mitigation strategy requires wisdom from leaders in making decisions on a priority scale for at-risk populations with limited resource allocation [6]. Another study revealed that assessing Resilience from a socialecological perspective in a community can be done using a quantitative approach [7]. There are have three fundamental problems in the Resilience of a community to reduce disaster risk, namely: preparation, response, and recovery, where the consequences of the approach affect (i) the relationship between communities, decisionmakers, and planning tools (ii) system capacity civil protection (iii) economic capacity[8].

Disasters that arise in a community will undoubtedly have an impact on the community itself. The resulting consequences often cause more harm than gain, so it takes time to recover from damage, disruption, and injury. Disaster resilience refers to a community's ability to withstand, adapt, and recover from disasters as quickly as possible and with little or no outside assistance $[9,10]$. As disasters often have a disproportionate impact on those with the poverty of technology and financial resources [11], the idea of Resilience should be more proactive to reduce disaster risk. Disasters that arise in a community will undoubtedly have an impact on the community itself. The effects often result in more harm than good, so it takes time to recover from damage, disruption, and injury. During the recovery period, a community's ability to withstand potential hazards is determined by the extent to which it possesses the necessary and systematic resources [12]. Amaratungga and Haigh [10] also recommend the need for early thinking about disaster risk reduction by increasing the roles and responsibilities of each stakeholder in increasing community resilience.

This paper's state of the art is the influence of the decision-making system on increasing the community's

\footnotetext{
* Corresponding author:putranesia@bunghatta.ac.id
} 
Resilience in disaster risk reduction efforts. This paper aims to determine the strength of a systematic decisionmaking support system in influencing community resilience in the face of disaster risk so that it can further make a more outstanding contribution to disaster risk reduction efforts in an appropriate and well-controlled manner.

\subsection{The Concept of Resilience}

Resilience can be defined as efforts in policy-making, framework creation, organizational/institutional, and community capacity (or non-structural actions) in disaster risk management [13]. Resilience can be described as a response to uncertainty from rapid and turbulent social change [14]. Another critical point is that the concept of Resilience recognizes community resilience through its capacity to self-regulate [15]. Resilience is a system that can be made if all subsystems and variables support the system, which can be measured by the extent to which the system can survive and absorb changes outside the system [16]. And also, according to Biesbroek, Dupuis, and Wellstead [17], Resilience has entered the public and political arena as a popular thing in viewing contemporary problems on issues of food security, climate change impacts, pollution, and natural resource management. The need to increase community resilience has become a sustainable development program (SDGs) in countries that are members of SDG 11. This is intended as an effort for Disaster Risk Reduction (DRR) in building resilient communities to reduce vulnerability to disasters Deep insight in realizing the community's unique personality, culture and understanding the mindset of the community at the individual perception level in adapting to increase their capacity to deal with disasters is needed to bridge the gap between spatial planning and disaster resilience building for disaster risk reduction in the future.

\subsection{Analysis of Decision Making in Resilience}

Successful decision-making in society begins with overcoming differences among community groups in decision-making [9]. As a result, decision-making must be based on uncertainty and incomplete knowledge for normative decisions based on the value of benefits (or utility) to be made by time systems, infrastructure systems, governance systems, regulatory systems, and risk systems [18]. On the other hand, Bhattacharya-Mis and Lamond [19] suggest that to increase Resilience, a realistic understanding of risk is needed to avoid external bias, where understanding risk will assist efforts in making the right decisions. In Disaster Risk Reduction, conceptually well-planned, adaptive, and sustainable planning will help policymakers avoid the worst outcome [20]. But in fact, Relative decision-making will differ on different dimensions of Resilience [21]. Due to the inconsistency of findings based on people who analyze data (subjective), a decision support system is hypothesized to reduce the amount of time needed to explore the data [22], allowing for faster and more accurate decisions. It's been shown that decision-making ability is influenced by the assessment of available resources and the decision-making power of policymakers [23]. This concept can reduce wrong decisions in overcoming problems in disaster risk reduction. The government, in this case, acts as a decision-maker that influences the six psychological constructs that will affect the performance of the construction (reconstruction) implementation, namely: culture, decision architecture, naturalistic decision making, group work, identity, and social representation [24]. Furthermore, Hettiarachchi and Weeresinghe [25] state that the primary measure for achieving disaster resilience is integrating Disaster Risk Reduction (DRR) into development at all levels.

\section{Methodology}

The research Methodology use "The Onion Proces" by Saunders [26].

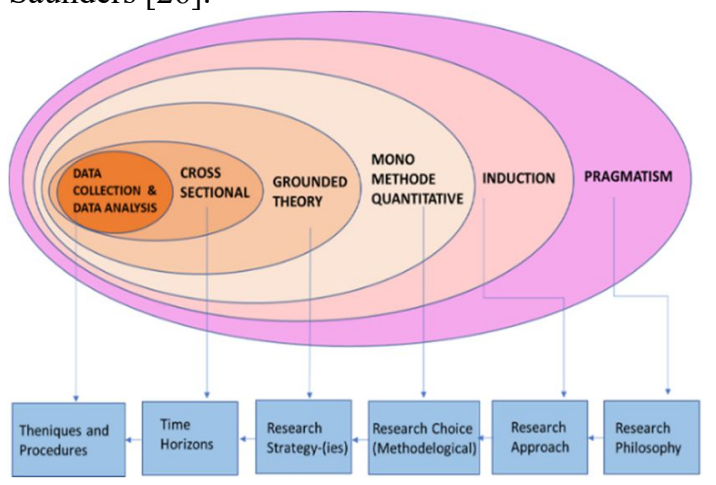

Fig.1. Developed From The Onion Proces by Saunders [26]

Fig. 1 explains that the research begins through a pragmatism approach by looking at the existing phenomena. Next, take an induction approach by exploring theories from previous researchers, which then go through a quantitative process to assess Resilience in a community from a socio-ecological perspective. Previous research was processed through a comprehensive literature review with data collection and analysis using the Feature Reporting Items for Systematic Review and Meta-Analysis (PRISMA).

\section{Framing Decision Support Systems in Resilience}

Recently, the concept of "resilience" has become a fundamental benchmark in various academic disciplines, including engineering research, organizational performance, ecological science, psychology, economics, climate change, disaster management, safety, security, and risk. Resilience appears to answer various problems and threats and thus attracts policymakers and researchers from multiple fields and disciplines. Policymakers are understood as people involved in organizational performance. Organizational Resilience can be understood as a learning organization that uncovers predictable and unpredictable disasters and is supported and nurtured as part of resilience goals [27]. Therefore, Resilience should be seen as an essential property of the system and not as an external quality [28]. Attempts to 
frame Resilience as a sustainability paradigm sometimes increase the complexity of decision making [29]. We must map the flow of information in reducing risk through collaboration, development planning and control, mitigation measures, building standards, community involvement, and effective management responses to assist all parties in making the right decisions [30], [31]. In information systems (IS), Decision Support Systems are used to support and improve managerial decisionmaking [32].
Learn from previous research to formulate a framework for decision-making support systems referring to disaster event data (big data) [33] and concrete steps in improving community resilience. Without the right decision support system, the amount of data will cause information overload [34]. Decision support system frameworks can be created based on hazards, resilience objectives, mitigation (ex-ante), and response (ex-post) strategies [35].

Fig. 2 explains the concept approach of Framing Decision Support Systems In Resilience

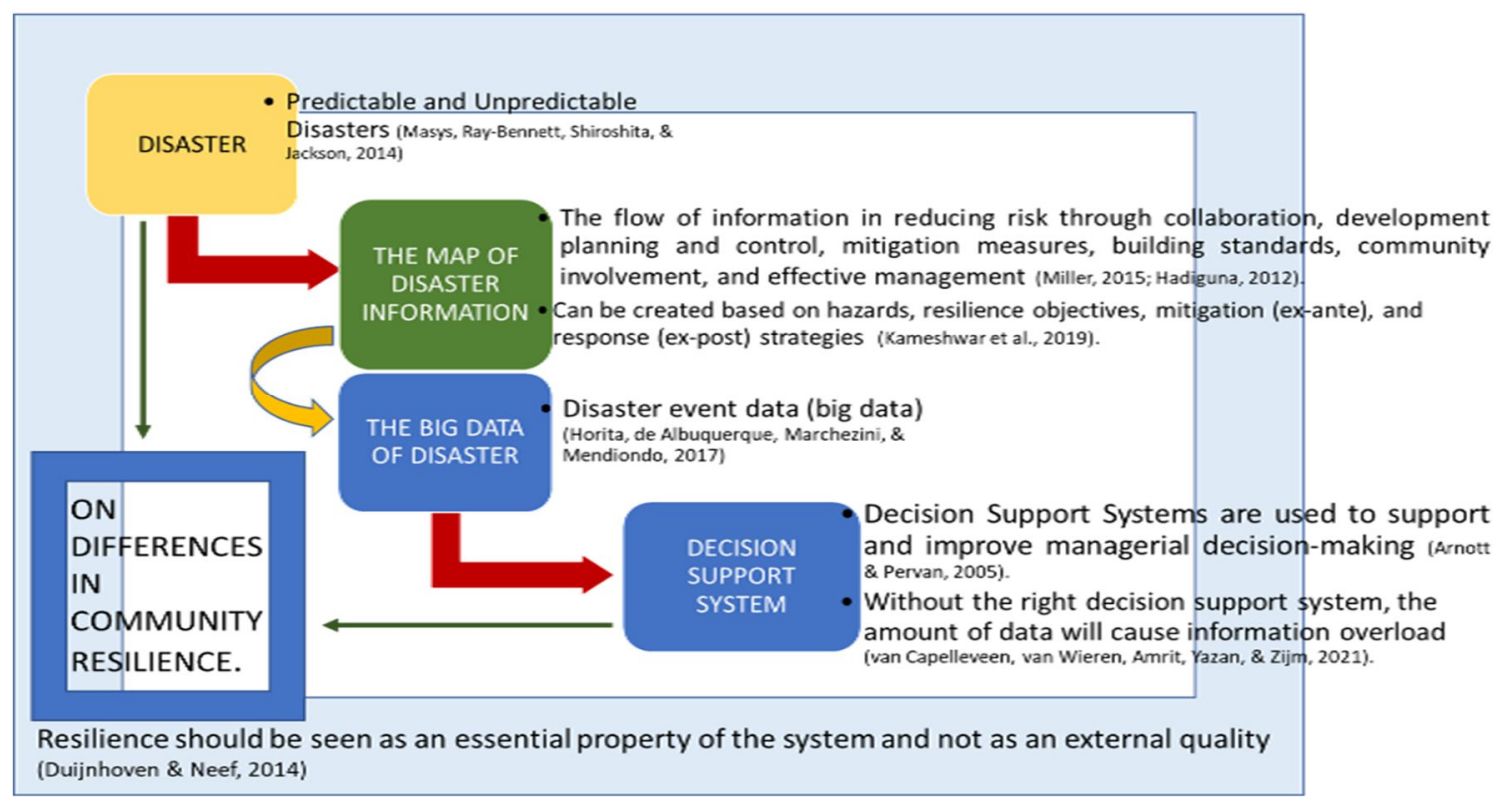

Fig.2. The Framing Decision Support Systems In Resilience [28]

\section{Conclusion}

As relative decision-making will differ in different dimensions of Resilience, a decision-making system will help provide effective decisions to reduce disaster risk in communities living in disaster-prone areas. In this regard, new space is obtained for further research related to decision-making. A decision support system with a good performance framework that is mixed based on differences in community resilience in disaster-prone areas can trigger disaster risk reduction of overall disaster resilience.

\section{References}

1 K. Reivich and A. Shatté, "The resilience factor: 7 essential skills for overcoming life's inevitable obstacles.," Resil. factor 7 Essent. Ski. overcoming life's inevitable Obs., 342, (2002)

2 V. Proag, "Assessing and Measuring Resilience," Procedia Econ. Financ., 18, September, 222-229, (2014)

3 H. Lindbom, H. Tehler, K. Eriksson, and T. Aven, "The capability concept - On how to define and describe capability concerning risk, vulnerability, and resilience," Reliab. Eng. Syst. Saf., 135,45-54, (2015)

4 K. Rus, V. Kilar, and D. Koren, "Resilience assessment of complex urban systems to natural disasters: A new literature review," Int. J. Disaster Risk Reduct.,. 31, January, 311-330, (2018)

5 T. R. H. Davies and A. J. Davies, "Increasing communities' resilience to disasters: An impactbased approach,” Int. J. Disaster Risk Reduct., 31, May, 742-749, (2018)

6 C. E. Kontokosta and A. Malik, "The Resilience to Emergencies and Disasters Index: Applying big data to benchmark and validate neighborhood resilience capacity," Sustain. Cities Soc., 36, June 2017, 272285, (2018)

7 W. C. Chuang et al., "Enhancing quantitative approaches for assessing community resilience," J. Environ. Manage., 213, 353-362, (2018)

8 M. Vona, M. Mastroberti, L. Mitidieri, and S. Tataranna, "New resilience model of communities based on numerical evaluation and observed postseismic reconstruction process," Int. J. Disaster Risk Reduct., 28, January, 602-609 (2018)

9 E. S. Ranjan and C. C. Abenayake, "A Study on Community's Perception on Disaster Resilience 
Concept," Procedia Econ. Financ., 18, September, 88-94, (2014)

10 C. Malalgoda, D. Amaratunga, and R. Haigh, "Challenges in Creating a Disaster Resilient Built Environment," Procedia Econ. Financ., 18, September, 736-744, (2014)

11 D. B. Oerther, "From disaster to development: Finance provides a platform to empower technology for resilience to climate change," Procedia Eng., 159, June, 267-271, (2016)

12 S. Platt, D. Brown, and M. Hughes, "Measuring resilience and recovery," Int. J. Disaster Risk Reduct., 19, May, 447-460, (2016)

13 K. MacAskill and P. Guthrie, "Multiple Interpretations of Resilience in Disaster Risk Management," Procedia Econ. Financ., 18, September, 667-674, (2014)

14 M. Iordan, M.-N. Chilian, and A. Grigorescu, "Regional Resilience in Romania - Between Realism and Aspirations," Procedia Econ. Financ., 22, November 2014, 627-635, (2015)

15 A. J. Imperiale and F. Vanclay, "Experiencing local community resilience in action: Learning from postdisaster communities," J. Rural Stud., 47, 204-219, (2016)

16 O. R. Danar and D. Pushpalal, "Building Community Resilience: Conceptual Framework and its Application in Post Tsunami Resettlement," Procedia Econ. Financ., 18, September, 489-496, (2014)

17 R. Biesbroek, J. Dupuis, and A. Wellstead, "Explaining through causal mechanisms: resilience and governance of social-ecological systems," Curr. Opin. Environ. Sustain., 28, 64-70, (2017)

18 M. H. Faber, L. Giuliani, A. Revez, S. Jayasena, J. Sparf, and J. M. Mendez, "Interdisciplinary Approach to Disaster Resilience Education and Research," Procedia Econ. Financ., 18, September, 601-609, (2014)

19 N. Bhattacharya-Mis and J. Lamond, "Socioeconomic Complexities of Flood Memory in Building Resilience: An Overview of Research," Procedia Econ. Financ., 18, no. September, 111-119, (2014)

20 K. Yang et al., "Hurricane evacuations in the face of uncertainty: Use of integrated models to support robust, adaptive, and repeated decision-making," Int. J. Disaster Risk Reduct., 36, October 2018, p. 101093 (2019)

21 C. W. Zobel and M. Baghersad, "Analytically comparing disaster resilience across multiple dimensions," Socioecon. Plann. Sci., 69, (2020)

22 R. S. Renu, G. Mocko, and A. Koneru, "Use of big data and knowledge discovery to create data backbones for decision support systems," Procedia Comput. Sci., 20, 446-453, (2013)

23 H. Lindbom, H. Hassel, H. Tehler, and C. Uhr, "Capability assessments - How to make them useful for decision-making," Int. J. Disaster Risk Reduct., vol. 31, no. May, pp. 251-259, (2018)

24 R. Turner, "Investigating how governmentality and governance influence decision making on projects," Proj. Leadersh. Soc., 1, May, p. 100003, (2020)

25 S. S. L. Hettiarachchi and S. Weeresinghe, "Achieving Disaster Resilience through the Sri Lankan Early Warning System: Good practices of Disaster Risk Reduction and Management," Procedia Econ. Financ., 18, September, 789-794, (2014)

26 A. Saunders; K .LEwis, Philip; Thornhill, Research Methods for Business Students, Eighth edi. New York : Pearson, [2019]: Print edition typeset in 9.5/12 ITC Slimbach Std by Pearson CSC, (2019)

27 A. J. Masys, N. Ray-Bennett, H. Shiroshita, and P. Jackson, "High Impact/Low-Frequency Extreme Events: Enabling Reflection and Resilience in a Hyper-connected World," Procedia Econ. Financ., 18, September, 772-779, (2014)

28 H. Duijnhoven and M. Neef, "Framing Resilience. From a Model-based Approach to a Management Process," Procedia Econ. Financ., 18, September, 425-430, (2014)

29 G. Lizarralde, K. Chmutina, L. Bosher, and A. Dainty, "Sustainability and resilience in the built environment: The challenges of establishing a turquoise agenda in the UK," Sustain. Cities Soc., 15, 96-104, (2015)

30 W. Miller, "What does built environment research have to do with risk mitigation, resilience, and disaster recovery?" Sustain. Cities Soc., 19, 91-97, (2015)

31 R. A. Hadiguna, "Decision support framework for risk assessment of sustainable supply chain," Int. J. Logist. Econ. Glob.,4, 1/2, 35, (2012)

32 D. Arnott and G. Pervan, "A critical analysis of decision support systems research," J. Inf. Technol., 20, 2, 67-87, (2005)

33 F. E. A. Horita, J. P. de Albuquerque, V. Marchezini, and E. M. Mendiondo, "Bridging the gap between decision-making and emerging big data sources: An application of a model-based framework to disaster management in Brazil," Decis. Support Syst., 97, 1222, (2017)

34 G. van Capelleveen, J. van Wieren, C. Amrit, D. M. Yazan, and H. Zijm, "Exploring recommendations for circular supply chain management through interactive visualisation," Decis. Support Syst., 140, 113431, (2021)

35 S. Kameshwar et al., "Probabilistic decision-support framework for community resilience: Incorporating multi-hazards, infrastructure interdependencies, and resilience goals in a Bayesian network," Reliab. Eng. Syst. Saf., 191, December, (2019) 